Elsevier required licence: (C) 2018

This manuscript version is made available under the CC-BY-NC-ND 4.0 license http://creativecommons.org/licenses/by-nc-nd/4.0/

The definitive publisher version is available online at https://doi.org/10.1016/i.asoc.2018.02.035 


\title{
Letter: The Concept of Stratification and Future Applications
}

\begin{abstract}
:
The main purpose of this letter is to draw attention to a recent concept, namely Concept of Stratification (CST) developed by Zadeh [1]. CST describes a system that transitions through a number of states in order to arrive at a desired state. CST is a problem-solving approach, which is easy while effective. Therefore, CST seems very likely to emerge in coming years as a major interest area in areas such as soft computing, Artificial Intelligence (AI), robotics, Natural Language Processing (NLP), and big data. In this expository letter, the advantages and the main shortcoming of CST are reviewed. The concept is explained and areas that the concept is likely to be applied are discussed. Considering the generality of the original CST proposed by Zadeh, it is possible to consider different versions for CST to be applied in future studies. Hence, versions of CST including fuzzy CST, a 3DCST, and multiple systems and multiple CSTs are presented. This work is a first step in a vast range of applications of CST. Researchers, especially those applying soft computing tools such as fuzzy sets theory and granulation, are encouraged to examine the capability of CST in addressing significant real-world problems.
\end{abstract}

Keywords: Concept of Stratification (CST), Fuzzy logic, Granulation; Artificial Intelligence (AI)

\section{Introduction}

Recently, a version of stratification, namely Concept of Stratification (CST), was proposed by Zadeh [1]. CST describes a system that transitions through a number of states in order to arrive at a desired state, namely a target state. The states, which have associated inputs and outputs, are incrementally stratified based on their distance from the target set. In comparison with the previous versions and applications of stratification, such as stratified logic [2], approach [3], programming [4], analysis [5], and others [6, 7], this new version of stratification, can handle a variety of problems yet is relatively easy to apply. The concept can be applied in soft computing, Artificial Intelligence (AI), robotics, Natural Language Processing (NLP) and many other areas. However, being a very new concept, further studies are needed to facilitate a full understanding of its value.

Admitting that there are similarities between CST and other approaches, such as the information granulation technique in soft computing (the process of decomposing the whole into parts) [8] or Breadth-First Search (BFS) algorithm [9], CST has its own characteristics. Although stratification can be considered as a type of granulation, CST is more than just a simple stratification [10]. CST considers a system, which based on the values of its variables, transitions toward the target set with a high level of dynamicity. Given the differences between CST and granulation, granular computing can be used in processing the variables of CST. In comparison with the BFS algorithm, CST includes an incremental enlargement process that follows an approach which is similar to the BFS algorithm. However, in contrast with BFS, CST is not focused on finding the shortest path to the target. Instead it uses a recursive approach of problem solving, concerned with finding a sequence of inputs that can transition a system from any state to a state in the target set.

The unique characteristic of CST is its very general and dynamic structure used to address real-world problems. Given the generality of the original CST, it can be easily extended and applied in future studies. The target set in CST is degraded, or incrementally enlarged, in order to perform the stratification process. Incremental enlargement provides CST with a high level of dynamicity [11]. This enlargement process enables CST to manage the stratification process and make it very similar to the process that happens in the human brain. For example, when we are asked to imagine a vehicle, then the request is refined to imagine a SUV vehicle, and after that, an automatic, white, SUV vehicle, our mind starts changing the precision of the image. From the first request, we imagine just a general and fuzzy picture of a vehicle. We do not consider the colour, the transmission system, the body type or other specifications of the vehicle. This is because the human brain does not bother with the consideration of unnecessary specifications and accuracy. However, hearing the second and third requests, the picture of the vehicle becomes more accurate and less fuzzy. Incremental enlargement performs a very similar process. While the primarily goal of such a target enlargement is to identify possible paths to reach the target, the enlargement serves as a semi optimization approach where: achieving a precise target is not required; is expensive or takes too much resources; and/or the target is currently ambiguous and becomes clear in the future. Such a useful approach in CST makes the concept suitable for its future vast applications.

Soft computing techniques, such as fuzzy logic [12], can be applied in combination with CST. For instance, fuzzy logic can be applied to linguistically define the inputs, outputs, and even the borders between the strata. Fuzzy definitions of such elements of CST extend the domain of applicability of CST to be utilised in many areas such as big data [13]. Empowering CST with methods, such as fuzzy logic, can stratify the data in a big 
data environment and present it to the user with respect to the user's target. This letter presents a number of extensions to CST such as Fuzzy CST (FCST), a 3D version of CST (3DCST), and Multiple Systems and Multiple CSTs (MSMCST) and suggests directions for future applications of CST. This work is a step to facilitate future applications of CST. To facilitate such applications, some potential extensions of CST are explained following a brief review of the original concept.

\section{A review of CST}

CST includes an underlying system, namely FSM, which transitions through strata. The strata are nested on the target set and include states. The system can pass the strata and move toward the target set by transitioning through the states. Every state includes state variables. The transitions are based on the value that the state variables have. Each state has inputs that can transition the system to different states. Therefore, there exists a sequence of inputs for each state, that if the system is provided with them, it is transitioned to a state in the target set $\left(\mathrm{T}_{0}\right)$. The target set can gradually be enlarged (incremental enlargement). The first enlarged target $\left(\mathrm{T}_{1}\right)$ or first stratum includes the target states plus all states which are only one step removed from the target states (Fig. 1). After the consideration of the first stratum as a part of the target set, the next stratum is identified using the same approach. In Fig. 1, the target set is at the bottom and includes two states initially. It starts the enlargement process by absorbing two states which are only one step removed from the target states. Through this, the first stratum is identified which includes four states. Following the same process, all of the states are stratified based on their distances from the target states. The grey arrow shows the incremental enlargement.

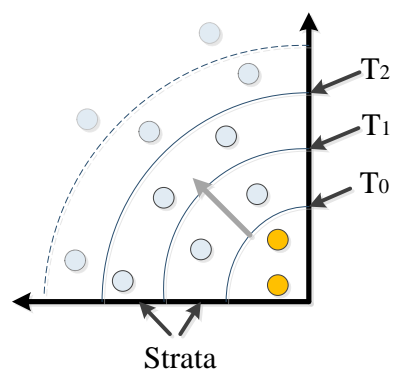

Figure 1. Strata, states, and the target set.

The following concepts are associated with CST:

- System: A system is a collection of objects that transitions through states of CST toward a state in the target set.

- State: A state, denoted by $\mathrm{w}_{\mathrm{i}}$, is identified with respect to the values of its associated variables. The state variables are predefined by the domain expert. The system transitions from one state to another, as the values of the variables change; the $i^{\text {th }}$ state is denoted as $\mathrm{w}_{\mathrm{i}}$.

- State-transition function: A state-transition function transitions the system from $\mathrm{i}^{\text {th }}$ state to $(i+1)^{\text {th }}$ state:

$$
\mathrm{s}_{(\mathrm{t}+1)}=\mathrm{f}\left(\mathrm{s}_{\mathrm{t}}, \mathrm{u}_{\mathrm{t}}\right)
$$

Explaining it more explicitly, each state can be considered as $s_{t}$ in order to find the next states that the system can transition to. Assuming that the system is at state $\mathrm{w}_{\mathrm{i}}$ (considered as $\mathrm{s}_{\mathrm{t}}$ ), it may receive an input, $\mathrm{u}_{\mathrm{t}}$, that transitions it from state $\mathrm{w}_{\mathrm{i}}$ to state $\mathrm{w}_{\mathrm{k}}$. In such a case, $\mathrm{w}_{\mathrm{k}}$ is a $\mathrm{s}_{(\mathrm{t}+1)}$ for $\mathrm{w}_{\mathrm{i}}$. This helps the identification of the existing paths from each state to other states.

- Inputs and outputs: There are one or more inputs $\left(\mathrm{u}_{\mathrm{t}}\right)$ for each state. Each input is associated with an output $\left(\mathrm{v}_{\mathrm{t}}\right)$ as follows.

$$
\mathrm{v}_{\mathrm{t}}=\mathrm{g}\left(\mathrm{s}_{\mathrm{t}}, \mathrm{u}_{\mathrm{t}}\right)
$$

- Target state: State $\mathrm{w}_{\mathrm{i}}$ is a target state if reaching $\mathrm{w}_{\mathrm{i}}$ is the objective of the system. This is the province of the system designer.

- Target set: If there are multiple target states for a system, they can be considered in a set of states, called target set. Note that CST can have more than one target states if the target states have the same value to the system.

- Stratum: Stratum $\mathrm{N}$ is a set of states from whcih a system can reach a target state in $\mathrm{N}$ or less than $\mathrm{N}$ steps.

- $\quad$ Reachability: $\mathrm{w}_{\mathrm{j}}$ is reachable from $\mathrm{w}_{\mathrm{i}}$ if there is a path between $\mathrm{w}_{\mathrm{i}}$ to $\mathrm{w}_{\mathrm{j}}$. 
CST and its main shortcoming are explained here through an example with only 6 states, in the interest of brevity.

Information Dominance (ID) is an operational advantage which is gained through the integration of information capability, systems, and resources in order to optimize decision making in the competitive environment [14]). Consider a system that has ID capability only in two states: $\mathrm{w}_{3}$ and $\mathrm{w}_{4}$, as shown in Table 1 . State $\mathrm{w}_{\mathrm{i}}$ is considered as a member of the target set, if the following proposition is true. $p=$ the system has an ID capability equal or above 0.95 .

Table 1. Input values for six states

\begin{tabular}{|c|c|c|c|c|}
\hline & Data quality & User involvement & Ease of navigation & ID capability \\
\hline $\mathrm{w}_{1}$ & 0.8 & 0.8 & 0.6 & 0.8 \\
\hline $\mathrm{w}_{2}$ & 0.85 & 0.85 & 0.7 & 0.9 \\
\hline $\mathrm{w}_{3}$ & 0.95 & 0.9 & 0.95 & 0.95 \\
\hline $\mathrm{w}_{4}$ & 0.95 & 0.9 & 0.9 & 0.95 \\
\hline $\mathrm{w}_{5}$ & 0.9 & 0.9 & 0.9 & 0.9 \\
\hline $\mathrm{w}_{6}$ & 0.85 & 0.85 & 0.9 & 0.8 \\
\hline
\end{tabular}

So, the target set in the example includes two states: $\left\{\mathrm{w}_{3}, \mathrm{w}_{4}\right\}$, as shown in Fig. 2.

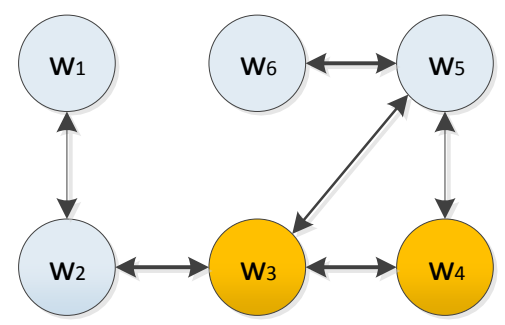

Figure 2. CST graph for ID capability

The states of the target set are reachable in one step, from states 2 and 5. Therefore, the incremental enlargement happens by absorbing $\mathrm{w}_{2}$ and $\mathrm{w}_{5}$ as the first stratum, and then states $\mathrm{w}_{6}$ and $\mathrm{w}_{1}$ as the second stratum. Through this, the stratification of the states is completed. The main shortcoming of CST appears when two or more objectives with different weights of importance are considered [10]. This has been admitted acknowledged by Zadeh [1] and is explained as follows. In the above example, consider two objectives. The first one is to achieve a state in $T_{1}:\left\{w_{3}, w_{4}, w_{5}\right\}$ and the second one is to achieve a state in $T_{2}:\left\{w_{2}, w_{3}, w_{4}\right\}$. The conjunction of the objectives is to achieve the intersection of the targets: $\mathrm{T}_{1} \cap \mathrm{T}_{2}:\left\{\mathrm{w}_{3}, \mathrm{w}_{4}\right\}$. If the importance weightings of both objectives were the same, the incremental enlargement would be the same as before: by absorbing $\left\{\mathrm{w}_{2}, \mathrm{w}_{5}\right\}$ and then $\left\{\mathrm{w}_{6}, \mathrm{w}_{1}\right\}$. However, the shortcoming appears when achieving one target (let's say target $\mathrm{T}_{1}$ ), is more important than the other target $\left(\mathrm{T}_{2}\right)$. In such situations, we cannot use stratification because we do not know how the incremental enlargement should be performed. This requires future study to develop an algorithm to address the enlargement process when the targets have unequal weights of importance.

\section{CST extensions}

Here, we provide three extensions to CST. The first one is when the power of fuzzy logic is leveraged to linguistically define the state variables, namely FCST. The second one is when a third dimension is taken into account for CST, namely 3DCST. The third one is when a number of systems are considered that can transition through states of different CSTs with the option of changing their CST at some states.

\subsection{Fuzzy CST (FCST)}

Utilising fuzzy logic in CST makes it more flexible to handle real-world problems. FCST can be developed with the consideration of fuzzy state variables, fuzzy inputs and (or) fuzzy outputs. The first one is explained here. In CST, a state is identified with respect to its associated variables. The system transitions from one state to another based on the input received in each state. The input changes the value of one or more variables. The change happens gradually rather than suddenly and hence fuzzy linguistic variables are used to describe this gradual change. This enables defining a finite number of predefined states. Thus, a system can be in more than one state at the same time with different membership functions. 
Consider a patient in a hospital. Variables like blood glucose and body temperature are measured and the values of state variables are defined based on the measurements. Consider the states for the patient/system with blood glucose as the only variable. Assume that the blood glucose is $260 \mathrm{mg} / \mathrm{dl}$. This can be considered as a state, and labelled state one. A few hours later, the blood glucose becomes 235. Now, the state can be labelled state two. Then, suppose that we are informed that the glucose has measured an hour ago by a nurse and it was 250 . Which state that would be? To answer such questions, either an infinite number of states must be considered, or else some linguistic intervals for the states. Fuzzy intervals enable the identification of the states that each patient belongs to when measuring the variables for the patient. The patient's blood glucose and body temperature are two variables which can be simply expressed as fuzzy linguistic variables. In the current example, blood glucose over 260 can be considered fully in interval one (very high), in which the patient is in an emergency situation and needs immediate treatment. If, for example, it drops below 230, it can be considered as having entered entirely into interval 2 (high) as presented in Fig. 3. For clarity, the transitions are illustrated by using fuzzy graphs for both state variables in Fig. 3 and 4.

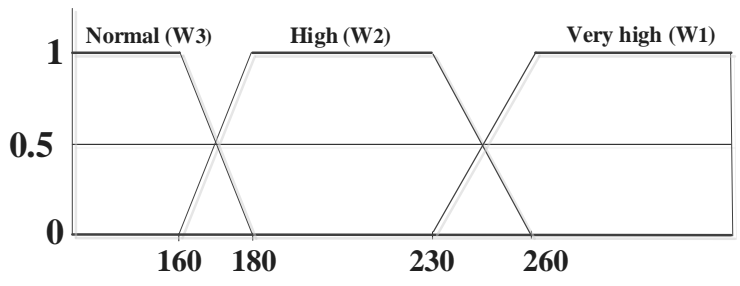

Figure 3. Blood glucose

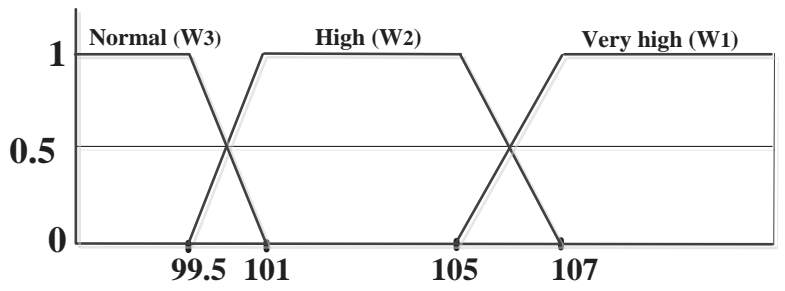

Figure 4. Body temperature $\left(F^{0}\right)$

Note that the number of states in CST is a multiplication of the numbers of the intervals that are considered for the state variables. In the current example, there are two state variables, blood glucose and body temperature, and each one has three intervals. Therefore, if the states are associated with these two variables, the number of states for the system would include nine states $(3 \times 3)$ : state 1 when 'blood glucose is very high and body temperature is very high', state 2 when 'blood glucose is very high and body temperature is high', and so on.

\subsection{DCST}

A system that transitions with one input $\left(\mathrm{u}_{\mathrm{t}}\right)$ in each state of CST can be presented as one-dimensional CST. If the system has two or more inputs, at least 2 dimensions are required to present CST. This, however, can be extended to a case where there are two or more inputs and one variable that has a unique nature. Based on the value that the variable receives, the system transitions to a parallel CST in which it is treated differently. Note that this can be extended to an ' $n$ ' dimensional CST. We present an example of 3DCST in this section.

In the patient example, imagine that besides from the patient's blood glucose and body temperature, there is a third variable, blood pressure, that is not being treated (no inputs). However, a change in the blood pressure can change the emergency level and hence the attention that the patient/system receives. If the blood pressure is very high, the patient moves to the third level of CST (L3: very high attention), if high, the second level of CST (L2: high attention), and if normal, the first level of CST (L1: normal attention) as shown in Fig. 5. 


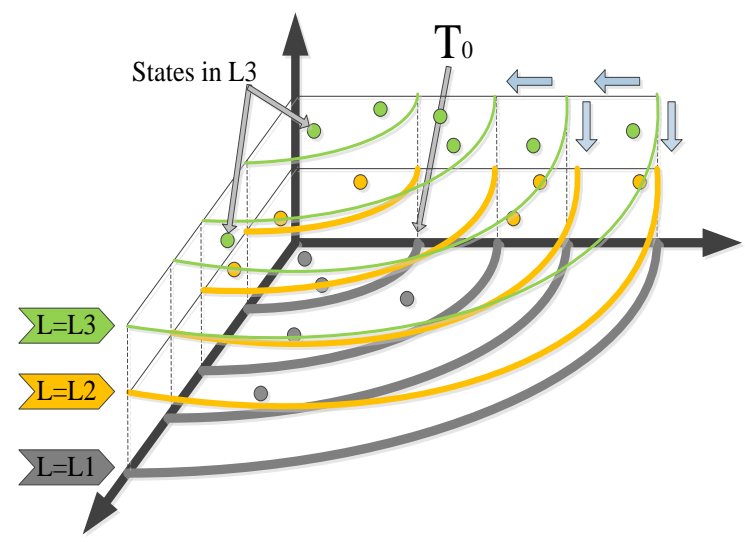

Figure 5: 3DCST for the patient example

In 3DCST, reaching to some certain states is impossible unless the system is transitioned to their certain level. The state space is defined as a finite set of states positioned on ' $\mathrm{n}$ ' levels, $\mathrm{W}$ : $\left(\mathrm{w}_{11}, \mathrm{w}_{12}, \mathrm{w}_{13} \ldots \mathrm{w}_{1 \mathrm{n}}, \mathrm{w}_{21 \ldots}, \mathrm{w}_{2 \mathrm{n}}, \ldots, \mathrm{w}_{\mathrm{m} 1}\right.$ $\left.\ldots . . \mathrm{w}_{\mathrm{mn}}\right)$. The following formula shows how 3DCST can be applied for a given problem where 'i' represents the discrete levels of importance of the system:

$$
\begin{aligned}
& \mathrm{s}_{(\mathrm{t}+1) \mathrm{i}}=\mathrm{f}_{\mathrm{i}}\left(\mathrm{s}_{\mathrm{ti}}, \mathrm{u}_{\mathrm{ti}}\right) \\
& \mathrm{v}_{\mathrm{ti}}=\mathrm{g}_{\mathrm{i}}\left(\mathrm{s}_{\mathrm{ti}}, \mathrm{u}_{\mathrm{ti}}\right)
\end{aligned}
$$

The output of each state is formulated as follows.

$$
v_{t i}=\left\{\begin{array}{cc}
a & \text { The patient is unhealthy } \\
b & \text { The patient is cured }
\end{array}\right\}
$$

Table 2 represents the results of the patient's examinations in 3DCST.

Table 2. The 3DCST for the patient example

\begin{tabular}{|l|c|c|c|c|c|}
\hline $\mathrm{S}_{\mathrm{t}}$ & $\begin{array}{c}\text { Blood } \\
\text { pressure }\end{array}$ & Temperature & $\begin{array}{c}\text { Blood } \\
\text { glucose }\end{array}$ & $\begin{array}{c}\text { Level of } \\
\text { importance }\end{array}$ & $\mathrm{v}_{\mathrm{ti}}$ \\
\hline $\mathrm{w}_{31}$ & $165 / 93$ & 40.5 & 130 & 3 & $\mathrm{a}$ \\
\hline $\mathrm{w}_{32}$ & $169 / 94$ & 41 & 127 & 3 & $\mathrm{a}$ \\
\hline $\mathrm{w}_{21}$ & $140 / 87$ & 38 & 115 & 2 & $\mathrm{a}$ \\
\hline $\mathrm{w}_{22}$ & $130 / 85$ & 39 & 110 & 2 & $\mathrm{a}$ \\
\hline $\mathrm{w}_{11}$ & $100 / 70$ & 37.5 & 90 & 1 & $\mathrm{~b}$ \\
\hline $\mathrm{w}_{12}$ & $125 / 82$ & 37 & 95 & 1 & $\mathrm{~b}$ \\
\hline $\mathrm{w}_{33}$ & $172 / 97$ & 39 & 119 & 3 & $\mathrm{a}$ \\
\hline $\mathrm{w}_{34}$ & $170 / 95$ & 41 & 126 & 3 & $\mathrm{a}$ \\
\hline
\end{tabular}

Based on Table 2, the eight states for the transitions of the system/patient in three levels can be visualised as shown in Fig. 6.

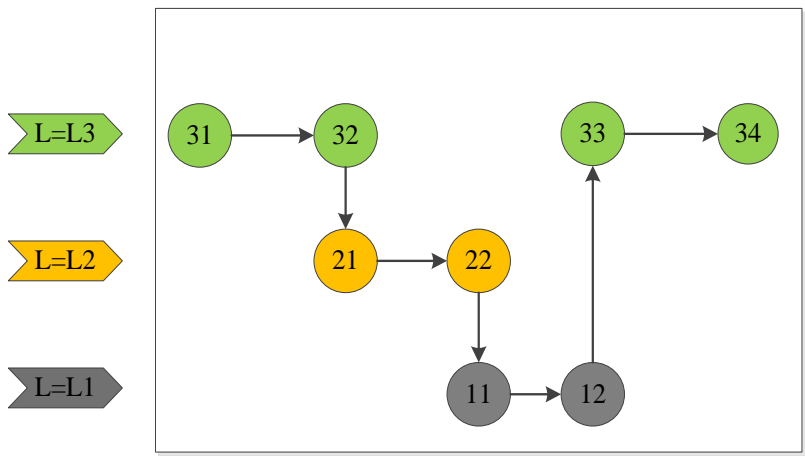

Figure 6. Eight states in 3DCST 
Note that another insight for the consideration of the third dimension can be new information. In the above example, imagine that the hospital receives information that the patient/system is the minister for education, a popular actor, a scientist, or even a spy. This information changes the level of attention that the system receives (the quality of the $\mathrm{u}_{\mathrm{t}}$ ). The information could be known to be incorrect during the transitions, so the level of attention changes again.

\subsection{Multiple Systems Multiple CSTs (MSMCST)}

Zadeh [1] describes one system that transitions on the states of a single CST. This can be generalised where there are multiple systems with the option of transitioning through multiple CSTs with different regulations, namely MSMCST. This has a great potential to be used for modelling real-world situations. For example, in a college, the systems are the students who have taken the same course. States are the sessions of the course. The output of each session can be measured in terms of students' level of learning. However, for different students, the journey to the target set can be different. The second CST, which some of these students could be transitioned to, is suspension CST with different states and regulations. A similar simulation can also be considered for many other situations such as in procurement processes with a number of potential suppliers. In MSMCST, the state transition and output functions are defined as follows.

$$
\begin{gathered}
{\left[s_{k p}, p\right]=f_{b j}\left(s_{t j}, u_{t b j}\right)} \\
v_{t b j}=g\left(s_{t j}, u_{t b j}\right) \\
\text { That: } \\
(k=t+1) \text { if and only if } p=j
\end{gathered}
$$

Function $\mathrm{f}_{\mathrm{bj}}$ transitions the system from $\mathrm{i}^{\text {th }}$ state to $(\mathrm{i}+1)^{\text {th }}$ state for $\mathrm{b}^{\text {th }}$ system in $\mathrm{j}^{\text {th }}$ CST or to $\mathrm{k}^{\text {th }}$ state in $\mathrm{p}^{\text {th }}$ CST.

The discussed themes can be employed in future studies on CST.

\section{Future directions}

There are many areas that CST can be applied in future studies. In particular, this letter encourages the application of CST in areas listed in the following section.

1) Robotics: With incremental enlargement, CST can enable robots to handle real-world situations, for instance the parking problem mentioned in previous papers $[15,16]$. While it is very easy for a human to park a vehicle, it is difficult for a robot to do the same. Although there are many activities that robots can perform much faster than humans, handling simple and routine activities: robots may be overwhelmed with numerous computations and calculations. Their inability to enlarge their initial target is one of the reasons that they fail to compete with humans in handling simple activities. Note that many decision making situations may require dealing with dynamic environments in which the goal/target, constraints and consequences of actions are inherently unknown [17].

2) Artificial Intelligence: One of the challenges in AI is the learning process [18]. For humans the level of attention given to detail is continuously changing. When a human hears or observes an event, the brain becomes alert depending on the level of importance of that event. Learning over a period while keeping the same level of attention is almost impossible. Most of the time, the learning target is enlarged and we only learn the main points without details. This saves the memory from being overwhelmed with unnecessary information. To mimic the human learning process, it is important to consider dynamicity in the amount of attention that is required to be given to detail.

3) Natural Language Processing (NLP): One of the achievements in NLP is to predict the next word while a person is writing something or correcting mistakes. Such predictions can be stratified and suggested depending on the user's proficiency level. Words can be positioned in strata. The user is provided with an option to determine the required level of accuracy. The more accurate the gauge, the fewer suggested options and the fewer the words suggested to the user. By enlarging the target, the user can have access to more predictive options. 
4) Big data: Big data is a buzzword which refers to the large volume of data, structured or unstructured, produced constantly by individuals and organizations. The data is stored in big databases, in various formats including texts, videos, and images. The unstructured formats make the analysis process more difficult than the traditional form of data which was usually structured. However, it is essential to utilise the data for achieving a higher quality decision making outcome. CST has the very good potential of managing big data. It can stratify the data and assist to handle its large size. The combination of fuzzy logic with CST can further stratify the data and compress it. Incremental enlargement provides the flexibility to analyse almost any system in a big data environment.

In addition, CST can be applied in future studies to address many problems that either could not be addressed using other methods or require complicated solutions. For instance, two decades ago Zadeh [16] discussed the fuzzy ball and beam problem. The problem is how to manage the beam to transfer the ball from one position to another specific position and keep it there (videos on the ball and beam problem are available on YouTube ${ }^{1}$ ). While Zadeh argues that fuzzy logic through soft computing and defining intervals can address this problem and save time and energy, he admits the incapability of fuzzy logic to consider the time dimension in computations saying "we do not yet completely understand how to apply fuzzy logic to problems like this "[16]. It is interesting that, now, after two decades, his new concept of CST can address this problem. The target position can be considered as the target set at time t. The next stratum includes the states which are only one step removed from the target. Being one step removed from the target means being in those states at time $t-1$ from where the target is reachable at time t. Following this process, all the possible strata and states around the target are identified. This approach helps to manage the forces needed to position the ball at the right time at any specific position. It can further identify if such a target is reachable considering the time limit and the available resources, and thus whether the enlargement of the target is essential or not. We believe that future studies will expose how CST can be profitable and provide easilty understandable solutions to many existing problems.

\section{Concluding remarks}

The main aim of this letter was to draw attention to a recent concept, namely CST. In summary, although there are similarities between CST and other approaches, such as the information granulation technique in soft computing or the BFS algorithm, CST has unique characteristics that provides the concept with flexibility to handle real-word problems. CST is equipped with the incremental enlargement process that is primarily used to identify possible paths to reach the target through states and then to find the sequence of inputs that transitions the system on the best path toward the target. Incremental enlargement can also be helpful where: achieving a precise target is not required; the specified target is expensive or takes too much time to reach; and/or the target is currently ambiguous and achieving its enlarged version is an alternative until it becomes clear in the future. The current version of CST can be extended for future applications. This study proposed three different extensions of CST, namely FCST, 3DCST and MSMCST which considerably facilitates future applications of CST. The main directions for future research on CST, including AI, robotics, NLP, and big data, were explained. We encourage researchers to examine the high capability of CST in addressing significant real-world problems.

\section{Acknowledgement:}

The letter was inspired by the one of the last contributions of a world-renowned scientist, Professor Lotfi Zadeh. Zadeh's theories of fuzzy logic have influenced many researchers in the last few decades, and we believe that CST also provides great potential for future research. We were shocked when we were informed recently that the world had lost one of its great researchers. May he rest in peace.

\section{References}

\footnotetext{
${ }^{1}$ https://www.youtube.com/results?search_query=ball+and+beam+problem
} 
1. Zadeh, L.A., Stratification, target set reachability and incremental enlargement principle. Information Sciences, 2016. 354: p. 131-139.

2. Zaniolo, C., Expressing and Supporting E ciently Greedy Algorithms as Locally Stratified Logic Programs. 2015.

3. Herencia, J., Graded sets and points: a stratified approach to fuzzy sets and points. Fuzzy Sets and Systems, 1996. 77(2): p. 191-202.

4. Dascalu, S.M., et al. Stratified Programming Integrated Development Environment (SPIDER). in IASSE. 2003.

5. Brenes, D.J. and D. Gayo-Avello, Stratified analysis of AOL query log. Information Sciences, 2009. 179(12): p. 1844-1858.

6. Balmin, A., et al., Stratified sampling using adaptive parallel data processing. 2017, Google Patents.

7. Cano, J.R., F. Herrera, and M. Lozano, On the combination of evolutionary algorithms and stratified strategies for training set selection in data mining. Applied Soft Computing, 2006. 6(3): p. 323-332.

8. Zadeh, L.A., Toward a theory of fuzzy information granulation and its centrality in human reasoning and fuzzy logic. Fuzzy sets and systems, 1997. 90(2): p. 111-127.

9. Kurant, M., A. Markopoulou, and P. Thiran. On the bias of bfs (breadth first search). in Teletraffic Congress (ITC), 2010 22nd International. 2010. IEEE.

10. Asadabadi, M.R., M. Saberi, and E. Chang, Targets of Unequal Importance Using the Concept of Stratification in a Big Data Environment. International Journal of Fuzzy Systems: p. 1-12.

11. Asadabadi, M., M. Saberi, and E. Chang, Logistic Informatics Modelling Using Concept of Stratification (CST). IEEE FUZZY 2017. IEEE Xplore., 2017.

12. Zadeh, L.A., Fuzzy logic= computing with words. IEEE transactions on fuzzy systems, 1996. 4(2): p. 103-111.

13. Wang, H., et al., A hybrid multi-objective firefly algorithm for big data optimization. Applied Soft Computing, 2017.

14. Endsley, M. and W.M. Jones, Situation Awareness Information Dominance \& Information Warfare. 1997, LOGICON TECHNICAL SERVICES INC DAYTON OH.

15. Zadeh, L.A., Fuzzy logic, neural networks, and soft computing. Communications of the ACM, 1994b. 37(3): p. 7785 .

16. Zadeh, L.A., Soft computing and fuzzy logic. IEEE software, 1994. 11(6): p. 48-56.

17. Zadeh, L.A., A note on Z-numbers. Information Sciences, 2011. 181(14): p. 2923-2932.

18. Asadabadi, M.R., M. Saberi, and E. Chang. A fuzzy game based framework to address ambiguities in performance based contracting. in Proceedings of the International Conference on Web Intelligence. 2017. ACM. 